\title{
Rendezvous at a Desired Point Irrespective of Agent Failures
}

\author{
Arpita Sinha \\ Systems and Control Engineering \\ Indian Institute of Technology, Bombay \\ Mumbai, India \\ Email: asinha@sc.iitb.ac.in
}

\begin{abstract}
In this paper, we study the rendezvous problem for a group of autonomous robots that can be terrestrial or aerial vehicles. The robots have to simultaneously meet at a point. This problem has been addressed by several researchers during the last few years. Various decentralized strategies have been developed and analyzed that guarantees rendezvous behavior. One such strategy is the cyclic pursuit strategy applied to the holonomic agents. Unlike most of the other strategies, cyclic pursuit allows to design the controller gain such that the agents meet at a desired point. However, if an agent fails, the cyclic connection between the agents will be lost and the whole system will break unless there is a rewiring of the connection and reassignment of controller gains. This becomes a non-trivial problem if we want to maintain the rendezvous point same as before. This paper elaborates a decentralized strategy that handles the issue of agent failure in cyclic pursuit. The rewiring mechanism to establish the cyclic connection and the reassignment of the gains are discussed and simulations are presented to validate the strategy presented in this paper.
\end{abstract}

\section{INTRODUCTION}

Research on cooperative control of multiple autonomous agents has been actively pursued during the last couple of years [1], [2]. Agents are the generic names for the autonomous vehicles like mobile robots, UAVs, under-water vehicles, and spacecrafts. A group of such agents are considered with each agent having limited resources and capabilities. The cooperative control strategy drives the agents to collectively achieve a goal which is beyond the capability of a single agent.

One of the interesting missions of the multi-agent systems is the cooperative rendezvous. Here, agents converge to a common point which may/maynot be prespecified. Bhattacharya et al. [3] studied the rendezvous problem of scalar agents having linear kinematics using ellipsoidal cone invariance properties. In [7] and [8], Lin et al. proposed a stop-and-go mechanism for rendezvous at an unspecified position by synchronous and asynchronous strategies. Rendezvous in a non-convex environment has been analyzed in [4]. Martinez [5] incorporated the measurement noise in the rendezvous problem. In [6], nonholonomic agents are considered and rendezvous in terms of both position and orientation is achieved.

Cyclic pursuit strategy has recently gather momentum in the the rendezvous and other formation control problems. Pavone et al. [10] achieved a circular and spiral formations using linear agents. In [12], a strategy to capture a target using cyclic pursuit is studied. Different geometric formations are obtained by Lin et al. [15]. Marshall et al. [14] analyzed both holonomic and nonholonomic agents. In [11], a smooth control law for two nonlinear agents under cyclic pursuit are studied. One of the initial work on cyclic pursuit was reported in [13]. In [9], heterogeneous agents are examined and the heterogeneity is utilized to obtain different behaviors - rendezvous at a prespecified position and directed motion.

In cyclic pursuit, the connection between the agents are cyclic and an agent follow another agent. Since there is only one leader for each agent, if one of the agent fails, the cyclic structure will break. Therefore, a rewiring is necessary. However, with rewiring the behavior of the agents will change from that of the original system. Here, we are interested to develop a strategy that ensure that the behavior of the system of agents remain as before even with an agent failure.

In this paper, the goal is that the agents should reach a prespecified location simultaneously. Each agent can only measure the distance of other agents with respect to itself and it is not aware of its own absolute position. We look at the control strategy developed in [9]. It has been shown in the paper that this problem can be solved by using linear cyclic pursuit, where the agent $i$ pursues agent $i+1$ (modulo $n$ ) and the controller gains of the agents are assigned based on the desired rendezvous point. Now, if one of the agents, say $p$, fails, the cyclic connection between the agents will be lost and the agent, that was following $p$, will not have anyone to pursue. Under this situation, it will be interesting to study how the cyclic connection can be established and the gains be reassigned so that the rendezvous point remains the same. The difficulty lies in the fact that there does not exist a leader or a centralized controller to solve this problem. Also, it will be advisable to have minimum amount of information exchange among the agents in order to tackle this situation in a decentralized manner. This paper addresses these issues and develops a decentralized strategy for the reassignment of the gains that guaranties that the goal is achieved.

This paper considers heterogeneous agents with linear kinematics. The main contribution of this paper is the contingency strategy in case of one or more agents failure. Section II discusses about the basics of linear cyclic pursuit. In Section III, the basic concepts about the contingency strategy is described. Section IV gives the methodology for implementation of the strategy. The decentralized algorithm is illustrated in Section 
V. In Section VI, simulations are presented and Section VII concludes the paper.

\section{PRELIMINARIES OF LINEAR CYCLIC PURSUIT}

Let us consider $n$ agents, ordered from 1 to $n$. Agent $i$ pursues agent $i+1$ modulo $n$. The agents start from any arbitrary positions on a plane. The position of the agents at time $t>0$ is given by

$$
Z_{i}(t)=\left[x_{i}(t), y_{i}(t)\right]^{T} \in \mathbb{R}^{2}, i=1,2, \ldots, n .
$$

The kinematic that models agent $i$ 's behavior, is

$$
\dot{Z}_{i}=u_{i}
$$

where $u_{i}$ is the control input given by

$$
u_{i}=k_{i}\left(Z_{i+1}-Z_{i}\right), \quad \forall i
$$

Thus, the velocity of the $i^{\text {th }}$ agent is proportional to the distance between the $i^{t h}$ and the $i+1^{t h}$ agent. The controller gain for agent $i$ is $k_{i}$.

Since, $x_{i}$ and $y_{i}$ coordinates of the $i^{t h}$ agent evolve independently for all $i$, they can be decoupled into two identical linear system of equations as

$$
\begin{aligned}
\dot{X}= & A X \\
A= & {\left[\begin{array}{cccccc}
-k_{1} & k_{1} & 0 & \ldots & \ldots & 0 \\
0 & -k_{2} & k_{2} & \ldots & \ldots & 0 \\
\vdots & \ddots & & & & \\
\vdots & & \ddots & & & \\
0 & 0 & 0 & \ldots & -k_{n-1} & k_{n-1} \\
k_{n} & 0 & 0 & \ldots & 0 & -k_{n}
\end{array}\right] }
\end{aligned}
$$

This system has been studied in details in [9]. Some of the results are presented here which will be applied directly in this paper.

The conditions for stability of (4) are given by the following theorem:

Theorem 1: Consider $n$ mobile agents with kinematics given by (4). For all initial conditions and for $k_{i}>0 \forall i$, all the agents converge to a point.

This is only a part of the stability results derived in [9]. In this paper, we will consider the controller gains to be positive and hence this result will be sufficient.

When the system is stable, the control law acts as a consensus strategy. The agents reach a consensus in position which implies the agents achieve rendezvous. The rendezvous point is a weighted average of the initial position of the agents and the weights depend on the gains of the agents. The next theorem gives an expression for the rendezvous point.

Theorem 2: If a system of $n$-agents having their initial positions at $Z_{i}\left(t_{0}\right)=\left[x_{i}\left(t_{0}\right), y_{i}\left(t_{0}\right)\right]^{T}$ and gains $k_{i}, \forall i$, satisfy Theorem 1 , then they converge to a point $Z_{f}$ given by,

$$
Z_{f}=\sum_{i=1}^{n}\left\{\left(\frac{1 / k_{i}}{\sum_{j=1}^{n} 1 / k_{j}}\right) Z_{i}\left(t_{0}\right)\right\}
$$

where $Z_{f}$ is called a reachable point for this system of $n$ agents.

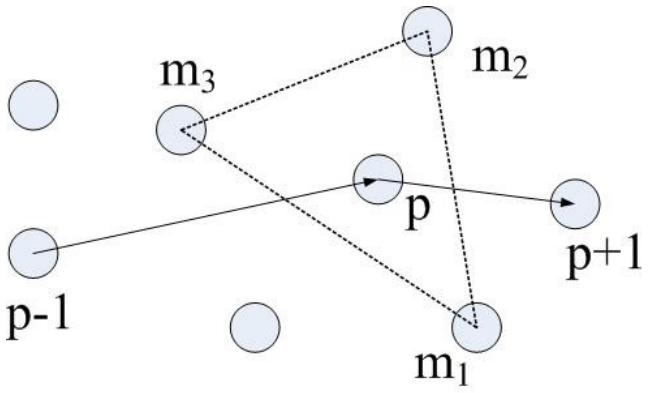

Fig. 1. Agents at $t=\tau$

The interesting point to be noted here is that for all time $t$

$$
Z_{f}=\sum_{i=1}^{n} \frac{Z_{i}(t) / k_{i}}{\sum_{j=1}^{n} 1 / k_{j}}
$$

which is stated in the proof of Theorem 2. This implies that the rendezvous point is the convex combination of the instantaneous position of the agents. The weights of the convex combination are functions of the controller gains. The gains of the controller can be designed such that the agents converge to a desired point. This result is detailed below:

Theorem 3: Consider a system of $n$ agents with initial positions at $Z_{i}\left(t_{0}\right), i=1, \cdots, n$. A point $Z$ is reachable if and only if,

$$
Z \in C o(S) \bigcup\left\{\bigcup_{p=1}^{n} C_{p}\right\}
$$

where $\operatorname{Co}(S)$ is the convex hull of $S=\left\{Z_{i}\left(t_{0}\right) \mid i=\right.$ $1,2, \cdots, n\}$ and $C_{p}$ is a cone that is finitely generated [16] by the vectors $\left(Z_{p}\left(t_{0}\right)-Z_{i}\left(t_{0}\right)\right), i=1, \cdots, p-1, p+1, \cdots, n$ and has vertex at $Z_{p}\left(t_{0}\right)$

This is a general result, but we are interested in $Z_{f} \in C o(S)$ since we consider positive gains of the controller. This result inspires us to address the problem that if an agent fails, the gains can be reassigned for the $n-1$ agents such that $Z_{f}$ remains the same. In the next section, the procedure for gain reassignment is discussed.

\section{CONTINGENCY STRATEGy}

In this section, we look at a strategy by which the agent $p$ can be replaced by other agents. We consider the position of the agents as points in a plane and derive all the analysis based on these points. Here, we look at the instant when the agent $p$ has failed.

Let us consider that at time $t=\tau$, agent $\mathrm{p}$ fails. The position of agent $\mathrm{p}$ at $\tau$ is given by $Z_{p}(\tau)$. From (7), we can write

$$
\sum_{i=1, i \neq p}^{n} \frac{Z_{i}(\tau) / k_{i}}{\sum_{j=1}^{n} 1 / k_{j}}+\frac{Z_{p}(\tau) / k_{p}}{\sum_{j=1}^{n} 1 / k_{j}}=Z_{f}
$$

that is, $Z_{f}$ is a convex combination of the points $Z_{i}(\tau), i=$ $1, \ldots, n$. The weights of each point is $\frac{1 / k_{i}}{\sum_{i=1}^{n} 1 / k_{i}}$. When the agent $p$ fails, we will have $Z_{p}(\tau)=0$. Therefore, the contribution by the point $Z_{p}(\tau)$ to obtain $Z_{f}$, which is equal 
to $\frac{Z_{p}(\tau) / k_{p}}{\sum_{j=1}^{n} 1 / k_{j}}$, has to be distributed among other points $Z_{i}(\tau)$. This requirement can be interpreted in another way. If we can express $Z_{p}(\tau)$ as a convex combination of some of the points $Z_{i}(\tau), i \neq p$, then the weight $\frac{Z_{p}(\tau) / k_{p}}{\sum_{j=1}^{n} 1 / k_{j}}$ can be shared by these points (refer to Figure 1). Considering the agents to be on a plane, then at least three such points are necessary.

Let us consider that $Z_{p}(\tau)$ can be represented as a convex combination of $Z_{m_{1}}(\tau), Z_{m_{2}}(\tau)$ and $Z_{m_{3}}(\tau)$, which are the positions of the agents $m_{1}, m_{2}$, and $m_{3}$ at $\tau$. Then, we can write

$$
\begin{aligned}
l_{m_{1}} Z_{m_{1}}(\tau)+l_{m_{2}} Z_{m_{2}}(\tau)+l_{m_{3}} Z_{m_{3}}(\tau) & =Z_{p}(\tau) \\
l_{m_{1}}+l_{m_{2}}+l_{m_{3}} & =1 \\
l_{m_{i}} \geq 0, \quad i=1,2,3 &
\end{aligned}
$$

The solution of (10)-(12) in term of the components along $x$ and $y$ coordinates can be obtained as

$$
\begin{aligned}
l_{m_{1}}= & \frac{1}{\Delta}\left(x_{m_{2}} y_{m_{3}}+x_{m_{3}} y_{p}+x_{p} y_{m_{2}}\right. \\
& \left.-x_{p} y_{m_{3}}-x_{m_{3}} y_{m_{2}}-x_{m_{2}} y_{p}\right) \\
l_{m_{2}}= & \frac{1}{\Delta}\left(x_{m_{1}} y_{p}+x_{p} y_{m_{3}}+x_{m_{3}} y_{m_{1}}\right. \\
& \left.-x_{m_{1}} y_{m_{3}}-x_{m_{3}} y_{p}-x_{p} y_{m_{1}}\right) \\
l_{m_{3}}= & \frac{1}{\Delta}\left(x_{m_{1}} y_{m_{2}}+x_{m_{2}} y_{p}+x_{p} y_{m_{1}}\right. \\
& \left.-x_{m_{1}} y_{p}-x_{p} y_{m_{2}}-x_{m_{2}} y_{m_{1}}\right)
\end{aligned}
$$

where

$$
\begin{aligned}
\Delta= & x_{m_{1}} y_{m_{2}}+x_{m_{2}} y_{m_{3}}+x_{m_{3}} y_{m_{1}} \\
& -x_{m_{1}} y_{m_{3}}-x_{m_{3}} y_{m_{2}}-x_{m_{2}} y_{m_{1}}
\end{aligned}
$$

Here, the positions $\left(x_{m_{i}}, y_{m_{i}}\right), i=1,2,3$, and $\left(x_{p}, y_{p}\right)$ are considered at $t=\tau$.

In terms of $l_{m_{1}}, l_{m_{2}}$ and $l_{m_{3}}$, (9) can be written as

$$
\begin{gathered}
\frac{\left(l_{m_{1}} Z_{m_{1}}(\tau)+l_{m_{2}} Z_{m_{3}}(\tau)+l_{m_{3}} Z_{m_{3}}(\tau)\right) / k_{p}}{\sum_{j=1}^{n} 1 / k_{j}} \\
+\sum_{i=1, i \neq p}^{n} \frac{Z_{i}(\tau) / k_{i}}{\sum_{j=1}^{n} 1 / k_{j}}=Z_{f}
\end{gathered}
$$

Rewriting the above equation

$$
\begin{gathered}
\frac{\left(1 / k_{m_{1}}+l_{m_{1}} / k_{p}\right) Z_{m_{1}}(\tau)}{\sum_{j=1}^{n} 1 / k_{j}}+\frac{\left(1 / k_{m_{2}}+l_{m_{2}} / k_{p}\right) Z_{m_{2}}(\tau)}{\sum_{j=1}^{n} 1 / k_{j}}+ \\
\frac{\left(1 / k_{m_{3}}+l_{m_{3}} / k_{p}\right) Z_{m_{3}}(\tau)}{\sum_{j=1}^{n} 1 / k_{j}}+\sum_{\substack{i=1,\left(i \neq p, m_{1}, m_{2}, m_{3}\right)}}^{n} \frac{Z_{i}(\tau) / k_{i}}{\sum_{j=1}^{n} 1 / k_{j}}=Z_{f}
\end{gathered}
$$

This equation should be true for all time $t \geq \tau$. Comparing the above equation with (9), we can see that the gains of agents $m_{1}, m_{2}$ and $m_{3}$ should be reassigned to $\bar{k}_{m_{1}}, \bar{k}_{m_{2}}$ and $\bar{k}_{m_{1}}$, where

$$
\frac{1}{\bar{k}_{m_{i}}}=\frac{1}{k_{m_{i}}}+\frac{l_{m_{i}}}{k_{p}}, \quad i=1,2,3
$$

The selection of the agents $m_{1}, m_{2}$ and $m_{3}$ will be discussed in the next section. Another point to be noted here is that to calculate $l_{m_{i}}, i=1,2,3$, we need to know the absolute position of the four agents $p, m_{1}, m_{2}$ and $m_{3}$. Given the type of problem we are addressing in this paper, it is not possible to obtain these absolute measurements. Hence, this problem has to be transformed such that reassignment of the gains can be done with the relative distance measurements. This will also be discussed in the next section.

Note: If $Z_{p}$ lies on the vertex of the convex hull of $Z_{i}(\tau), \forall i$, then we can not represent $Z_{p}(\tau)$ as a convex combination of the points $Z_{i}(\tau), \forall i \neq p$. Therefore, this strategy of replacing the failed agent $p$ cannot be applied. However, we may still solve (10) and (11). The solution will contain $l_{m_{i}}<0$ for some $i$. The new gains will be computed using (19). If the gains are all positive, our objective will be satisfied. If not, we will have to find suitable candidate agents for replacement such that the final rendezvous point is very close to $Z_{f}$. We intend to address this problem in details in a subsequent paper and here we will assume that agent $p$ is not at the vertex of the convex hull of all the agents at $t=\tau$.

\section{GAIN REASSIGNMENT}

In this section, we address two problems. The first problem is to find the agents $m_{1}, m_{2}$ and $m_{3}$ such that agent $p$ is within the convex hull formed by these agents. In the second problem, we discuss how to calculate the new gains for the agents $m_{1}, m_{2}$ and $m_{3}$ when only the relative position of the agents can be measured.

Let us first look at the problem of how to find the new gains of the agents $m_{1}, m_{2}$ and $p-1$.

\section{A. Gain calculation}

The new gains of the agents $m_{1}, m_{2}$ and $m_{3}$ are given by (19). In order to evaluate (19), the agents have to calculate $l_{m_{i}}, i=1,2,3$, which are given in (13)-(15). We can rewrite these equations as

$$
\begin{aligned}
& l_{m_{1}}= \\
& \frac{\left(x_{p}-x_{m_{3}}\right)\left(y_{m_{2}}-y_{m_{3}}\right)-\left(x_{m_{2}}-x_{m_{3}}\right)\left(y_{p}-y_{m_{3}}\right)}{\left(x_{m_{1}}-x_{m_{3}}\right)\left(y_{m_{2}}-y_{m_{3}}\right)-\left(x_{m_{2}}-x_{m_{3}}\right)\left(y_{m_{1}}-y_{m_{3}}\right)} \\
& l_{m_{2}}= \\
& \frac{\left(x_{m_{1}}-x_{m_{3}}\right)\left(y_{p}-y_{m_{3}}\right)-\left(x_{p}-x_{m_{3}}\right)\left(y_{m_{1}}-y_{m_{3}}\right)}{\left(x_{m_{1}}-x_{m_{3}}\right)\left(y_{m_{2}}-y_{m_{3}}\right)-\left(x_{m_{2}}-x_{m_{3}}\right)\left(y_{m_{1}}-y_{m_{3}}\right)} \\
& l_{m_{3}}=\quad \nu \\
& \frac{\nu}{\left(x_{m_{1}}-x_{m_{3}}\right)\left(y_{m_{2}}-y_{m_{3}}\right)-\left(x_{m_{2}}-x_{m_{3}}\right)\left(y_{m_{1}}-y_{m_{3}}\right)}
\end{aligned}
$$

where

$$
\begin{aligned}
\nu= & \left\{\left(x_{m_{2}}-x_{m_{3}}\right)-\left(x_{m_{1}}-x_{m_{3}}\right)\right\} \\
& \left\{\left(y_{p}-y_{m_{3}}\right)-\left(y_{m_{1}-y_{m_{3}}}\right)\right\}-\left\{\left(x_{p}-x_{m_{3}}\right)-\right. \\
& \left.\left(x_{m_{1}}-x_{m_{3}}\right)\right\}\left\{\left(y_{m_{2}}-y_{m_{3}}\right)-\left(y_{m_{1}}-y_{m_{3}}\right)\right\}
\end{aligned}
$$

It can be seen that $l_{m_{1}}, l_{m_{2}}$ and $l_{m_{3}}$ can be computed from the relative distances of the agents $p, m_{1}$ and $m_{2}$ from the agent $m_{3}$ at time $t=\tau$. 


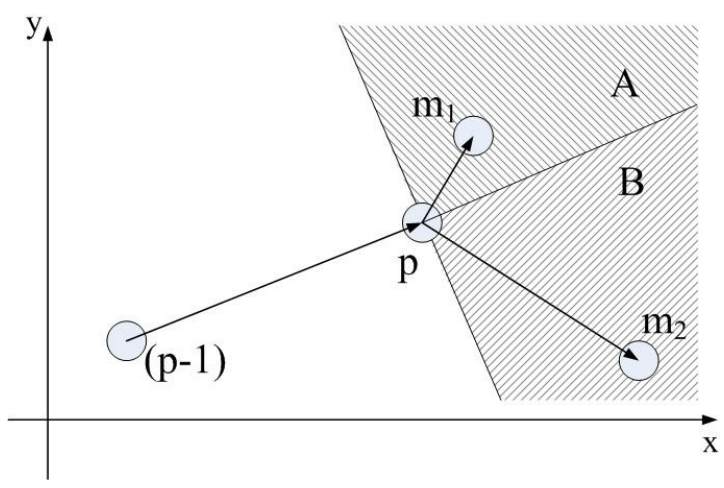

Fig. 2. Agent selection

Now, agent $p$ is pursued by agent $(p-1)$. When agent $p$ fails, only agent $(p-1)$ knows $\left(Z_{p}(\tau)-Z_{p-1}(\tau)\right)$. Therefore, it becomes obvious to consider agent $m_{3}$ as the agent $(p-1)$. It then remains to find the agents $m_{1}$ and $m_{2}$ and their relative distances from agent $(p-1)$.

\section{B. Agent selection}

In order to replace agent $p$ that failed at time $\tau$, we need three agents $m_{1}, m_{2}$ and $m_{3}$ such that the agent $p$ is within the convex hull formed by the agents $m_{i}, i=1,2,3$ at $t=\tau$. We assumed that the agent $p-1$ is agent $m_{3}$ as discussed in the previous subsection. The selection of the other two agents $m_{1}$ and $m_{2}$ can not be done independently. However, if the number of agents are sufficiently large, then we propose a mechanism by which the agents $m_{1}$ and $m_{2}$ can be independently selected. Here, we do not quantify the term "large", but assume that when it is large, the proposed method will work under most of the situations. In Section VI, Monte Carlo simulations are carried out to show how the number of agents affects the success of this algorithm.

Consider Figure 2 which shows the agents $p$ and $p-1$ at $t=\tau$. We can divide the plane into four quadrants by the line $\overrightarrow{Z_{p} Z_{p-1}}$ and its perpendicular. Two of the quadrants are marked $A$ and $B$ and are shaded in the Figure 2. The objective is to select one agent from each of the two sectors. This will ensure that the agent $p$ remains within the convex hull of the selected agents, $m_{1}$ and $m_{2}$ and the agent $p-1$. This is a restrictive condition but it ensures that the selection of $m_{1}$ and $m_{2}$ do not depend on each other. However, it may happen that either or both the sectors do not contain any agents. If there are no agents in both the sectors, it implies that the agent $p$ is at the vertex of the convex hull of all the $n$ agents. This condition is mentioned in the note of the previous section. On the other hand, if one of the sector is empty, the present method can be extended. We will not address this problem in this paper due to the page limitation.

Consider the sector $A$. An agent $m_{1}$ in this sector should satisfy both the following conditions

$$
\begin{array}{r}
{\left[\begin{array}{l}
x_{m_{1}}-x_{p} \\
y_{m_{1}}-x_{p}
\end{array}\right]^{T}\left[\begin{array}{c}
x_{p}-x_{p-1} \\
y_{p}-y_{p-1}
\end{array}\right]} \\
{\left[\begin{array}{c}
x_{m_{1}}-x_{p} \\
y_{m_{1}}-x_{p}
\end{array}\right]^{T}\left[\begin{array}{c}
-\left(y_{p}-y_{p-1}\right) \\
x_{p}-x_{p-1}
\end{array}\right] \geq 0}
\end{array}
$$

We will similarly have a set of conditions for sector $B$. Agent $(p-1)$ can evaluate these conditions since it can calculate $Z_{m_{1}}-Z_{p}=\left(Z_{m_{1}}-Z_{p-1}\right)-\left(Z_{p}-Z_{p-1}\right)$. Therefore, agent $(p-1)$ will be able to select agents $m_{1}$ and $m_{2}$.

Remark: Two of the three agents $m_{i}, i=1,2,3$ are collinear with agent $p$. This corresponds to the equality in either of (23) and (24) or its counterpart in the other sector. This implies that the agent $p$ lies on the boundary of the convex hull of $m_{i}$, $i=1,2,3$ and therefore one of the weights $l_{m_{i}}$ will be zero.

The next section will consolidate the procedure developed for replacement of a failed agent in a decentralized setup.

\section{DECENTRALIZED IMPLEMENTATION}

In this section, we elaborate the decentralized strategy that needs to be invoked when an agent fail. We make the following assumptions -

(a) a cyclic connection exists between the agents where agent $i$ follows $i+1$, modulo $n$

(b) controller gain $k_{i}$ for all $i$ is decided a priori based on the desired rendezvous point

(c) each agent $i$ knows the gains $k_{i}$ and $k_{i+1}$

(d) each agent $i$ knows the identity of agent $i+2$ (leader of $i+1)$ and $i-1$ (follower of itself).

When an agent $p$ fails, agent $p-1$ has a crucial role to play. In order to retain the desired rendezvous point, agent $p-1$ and two more agents has to change their gains. Agent $p-1$ selects an agent $m_{1}$ that lies in the sector $A$ of Figure 2. It also selects an agent $m_{2}$ from the sector $B$. It establishes a communication link with the agents $m_{1}$ and $m_{2}$, and obtains the value of their gains $k_{m_{1}}$ and $k_{m_{2}}$. Since agent $p-1$ can sense the relative distance of agents $m_{1}$ and $m_{2}$ with respect to itself and it knows the relative distance to agent $p$, it can compute the new gains $\bar{k}_{m_{1}}, \bar{k}_{m_{2}}$ and $\bar{k}_{p-1}$ using (19). Agent $p-1$ communicates the new gains to the agents $m_{1}$ and $m_{2}$ which they start using immediately in their control law. Also agent $p-1$ starts using the new gain.

Secondly, to reestablish the cyclic connection between the agents, agent $p-1$ should start to follow agent $p+1$. Since the identity of agent $p+1$ was available to agent $p-1$, it can search and start following $p+1$. However, the rewiring should be informed to the agents $p-2$ and $p+2$ as given in assumption (d) above. This can be done by transmitting the information along the cyclic connection.

This algorithm is illustrated in Table I. The steps 1-8 have to be completed as quickly as possible, while step 9-11 can tolerate some delays. Our assumption here is that the time required to complete the steps $1-8$ will be such that the distances moved by the agents are not significant compared to the total distance they have to cover. This is a decentralized 


\section{ALGORITHM}

(executed by agent $(p-1)$ when agent $p$ fails)

\begin{tabular}{|c|c|}
\hline ep 1: & Select agent $m_{1}$ that satisf \\
\hline 2: & agent $m_{2}$ \\
\hline p 3: & ntact with $m_{1}$ and $m_{2}$ \\
\hline ep 4: & $\begin{array}{l}\text { Obtain the gains } k_{m_{1}} \text { and } k_{m_{2}} \text { from agents } m_{1} \\
\text { and } m_{2} \text { respectively }\end{array}$ \\
\hline ep 5: & Compute $l_{m_{i}}, i=1,2,3$ from (20)-(22) \\
\hline 6: & Com \\
\hline ep 7: & cate $\bar{k}_{m_{1}}$ and $\bar{k}_{m_{2}}$ to $m_{1}$ and $m_{2}$ re- \\
\hline tep & Sear \\
\hline tep 9: & the identity of agent \\
\hline tep 10: & Inicate to agent $p+1$ that it is following \\
\hline ep 1 & $\begin{array}{l}\text { Obtain the identity of agent } p+2 \text { and gain } k_{p+} \\
\text { from agent } p+1\end{array}$ \\
\hline
\end{tabular}

TABLE I

strategy where only three agents are involved. We require three agents because we are considering a two-dimensional plane where the agents are moving. If the agents are restricted to onedimensional plane, only two agents will be necessary, while in the case of three-dimension, four agents will be required. We can follow a similar procedure for selecting the agents and changing their gain values.

Remark: It is to be noted here that in order to calculate $l_{m i}$ for $i=1,2,3$ in (13)-(15), the $x$ and $y$ coordinates of the position of agents $m_{1}$ and $m_{2}$ are required. However, the agent $p-1$ does not have to maintain a global reference frame for this purpose since (10)-(12) are invariant under rotation of the reference frame.

In the next section, we demonstrate this strategy on a group of agents moving on a plane.

\section{Simulation RESUlTS}

First, we illustrate the gain reassignment strategy for particular case and then show the Monte carlo simulation for different initial conditions. Consider ten agents randomly placed in a two-dimensional plane. The gains of the agents are also selected randomly. The position and the gains are tabulated in Table II. When non of the agents fail, they rendezvous at $(-20.8-22.6)$. This is shown in Figure 3 .

In the next simulation, the agent Red fails after $0.1 \mathrm{sec}$ from start. The cyclic connection of the agents are such that the Blue follows the Red, the Red follows the Green and the Green follows the Yellow. Therefore, Blue should now follow Green. In 0.1 second, the agents have traced a trajectory given by the red lines in Figure 4. When the Red fails, blue selects Green and Yellow to change their gains. It is by coincidence that Blue has selected Green which is also the agent that Blue has to follow. The new gains for the Blue, Green and Yellow agents

\begin{tabular}{|l|l|l|l|l|}
\hline Agent & Position at $t=0$ & $\begin{array}{l}\text { Position at } t= \\
0.1\end{array}$ & $\begin{array}{l}\text { Initial } \\
\text { gains }\end{array}$ & $\begin{array}{l}\text { New } \\
\text { gains }\end{array}$ \\
\hline 1 & $(-3.7,-38.2)$ & $(-12.72,-41.23)$ & 0.66 & 0.66 \\
2 & $(-160.3,-95.3)$ & $(-131.43,-75.70)$ & 1.66 & 1.66 \\
3 & $(33.9,23.3)$ & $(25.41,39.70)$ & 2.66 & 2.66 \\
4 & $(-13.1,123.5)$ & $(5.78,69.77)$ & 3.66 & 3.66 \\
5 & $(48.5,-57.8)$ & $(46.87,-44.43)$ & 4.66 & 4.66 \\
6 & $(59.9,-50.1)$ & $(33.58,-3.24)$ & 5.66 & 5.66 \\
Blue & $(-8.6,72.3)$ & $(1.09,50.81)$ & 6.66 & 3.80 \\
Red & $(32.5,3.9)$ & $(-1.10,28.10)$ & 7.66 & - \\
Green & $(-33.5,154.1)$ & $(-27.01,-3.31)$ & 8.66 & 9.10 \\
Yellow & $(-32.2,-170.1)$ & $(-17.94,-89.59)$ & 9.66 & 7.88 \\
\hline
\end{tabular}

TABLE II

Positions AND GAINS OF THE AGENTS

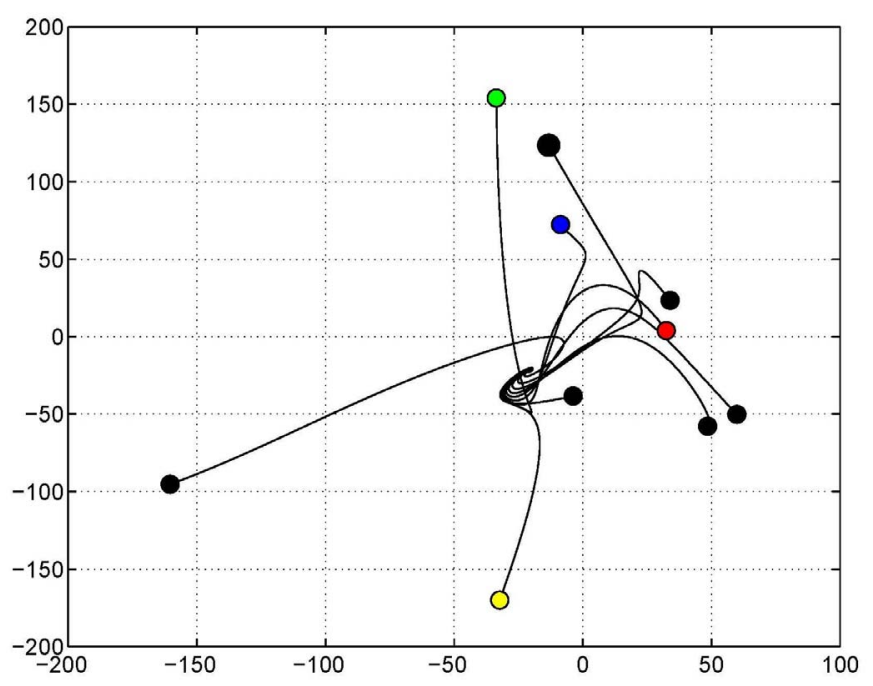

Fig. 3. Trajectory of 10 agents

are shown in the Table II. The nine agents finally converge to the point $(-20.8-22.6)$ which is the rendezvous point of the original systems with ten agents. The trajectories of the agents are shown in Figure 4.

However, consider the case when the agents did not modify their gains but established the cyclic connection. It can be seen from simulation that when Red fails and Blue starts following Green while the gains of all the agents remain the same, the rendezvous point is $(-21.59,-24.48)$. This is different from the one obtained when there is no failure.

To test the success rate of this algorithm, a Monte Carlo simulation is generated which is shown in Figure 5. Each bar corresponds to the size of the group of agents, which is varied from group of 10 agents to a group of 200 agents. For each group, simulations are carried out for 100 different initial conditions. In all the cases, we considered that the agent 1 has failed at $0.1 \mathrm{sec}$. The plots in the figure shows the number of times the algorithm could successfully resign the gains. It can be seen that as the group size increases, that is, the number 


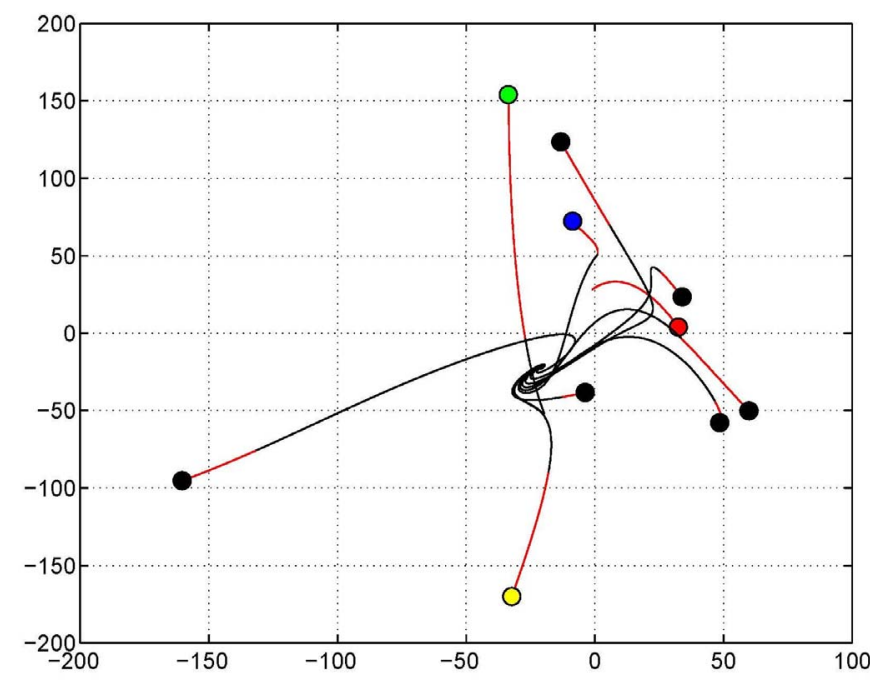

Fig. 4. Trajectory of 10 agents: Red agent fails

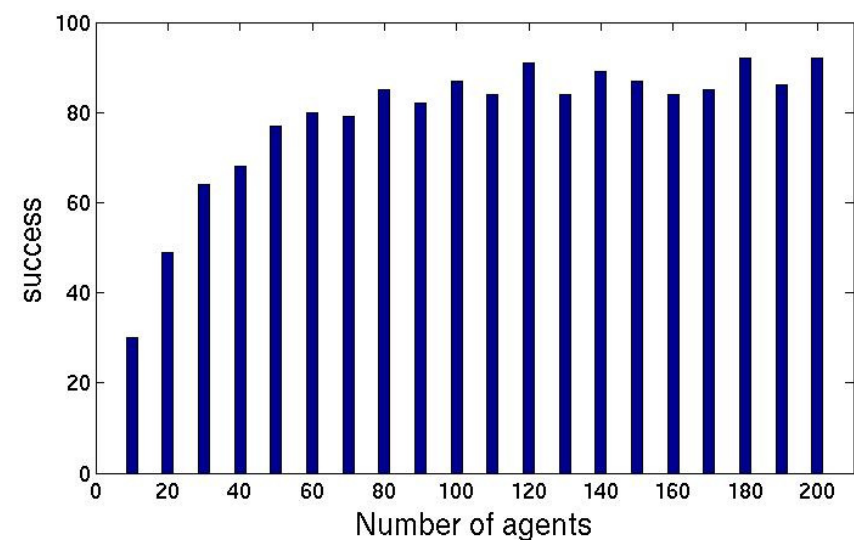

Fig. 5. Rate of success for 100 initial conditions

of agents increases the higher is the rate of success of the algorithm.

\section{CONCLUSION}

In this paper, we studied the case when there is an agent failure in a rendezvous problem. The group of agent moving under cyclic pursuit are supposed to rendezvous at a desired point $Z_{f}$. If during the mission, one of the agent fails, it is still desirable that the rest of the agents converge to $Z_{f}$. However, we assume that the agents cannot measure their absolute positions and their control actions are based on the relative distance between the agents. The rendezvous point is a function of the agents' gains and the gains are modified online so that the rendezvous point does not change. In this paper, a decentralized strategy is proposed that requires only three agents to modify their gains. The agent that has failed must be within the convex hull of the three agents. The new gains are calculated from the relative distances between the agents. The communications and computations are simple and hence it is assumed that the new gains can be calculated before any significant movement of the agents. It is also assumed that the three agents involved in gain rescheduling will not fail until the process is complete. This is a reasonable assumption since we so not expect the agents to fail very frequently. The behavior of the rest of the agents remain unchanged. The theoretical results are verified though simulations.

However, new strategy can be developed when we can not find three agents, the convex hull of which contains the failed agent. Also, the systems with negative controller gains will be interesting to address. A future extension of this work will be to apply this concept for general connection topologies.

\section{REFERENCES}

[1] R.M. Murray: "Recent research in cooperative control of multivehicle systems", ASME Journal of Dynamic Systems, Measurement, and Control, Vol 129, No 5, 2007, pp. 571-583.

[2] W. Ren, R.W. Beard, E.M. Atkins: "A survey of consensus problen in multi-agent coordination", Proceedings of the American Control Conference, Boston, Massachusetts, USA, June 2004, pp. 1859 - 1864.

[3] R. Bhattacharya, A. Tiwari, J. Fung, R.M. Murray: "Cone invariance and rendezvous of multiple agents", Proceedings of the Institution of Mechanical Engineers, Part G: Journal of Aerospace Engineering, Vol 223, No 6, 2009, pp. 779-789

[4] A. Ganguli, J. Cortes, and F. Bullo: "Multirobot rendezvous with visibility sensors in nonconvex environments", IEEE Transactions on Robotics, Vol 25, No 2, 2009, pp. 340-352

[5] S. Martinez: "Practical multiagent rendezvous through modified circumcenter algorithms", Automatica, Vol 45, No 9, 2009, pp. 2010-2017

[6] D.V. Dimarogonas and K.J. Kyriakopoulos: "On the rendezvous problem for multiple nonholonomic agents", IEEE Transactions on Automatic Control, Vol 52, No 5, 2007, pp. 916-921.

[7] J. Lin, A.S. Morse, B.D.0. Anderson: "The multi-agent rendezvous problem. Part 1: The synchronous case", SIAM Journal on Control and Optimization, Vol 46, No 6, 2007, pp. 2096-2119.

[8] J. Lin, A.S. Morse, B.D.0. Anderson: "The multi-agent rendezvous problem. Part 2: The asynchronous case", SIAM Journal on Control and Optimization, Vol 46, No 6, 2007, pp. 2120-2147

[9] A. Sinha and D. Ghose: "Generalization of linear cyclic pursuit with application to rendezvous of multiple autonomous agents", IEEE Transactions on Automatic Control, Vol 51, No 11, 2006, pp. 1819-1824.

[10] M. Pavone and E. Frazzoli: "Decentralized policies for geometric pattern formation and path coverage", ASME Journal of Dynamic Systems, Measurement, and Control, Vol 129, No 5, 2007, pp. 633-643.

[11] S. Zhao and T. Kalmar-Nagy: "Nonlinear dynamics of uni-cyclic pursuit", IEEE International Symposium on Intelligent Control, San Antonio, Texas, USA, September 2008, pp. 395-400

[12] T. Kim and S. Hara: "Stabilization of multi-agent dynamical systems for cyclic pursuit behavior", Proceedings of the IEEE Conference on Decision and Control, Cancun, Mexico, Dec 2008, pp. 4370-4375

[13] A.M. Bruckstein, N. Cohen and A. Efrat: "Ants, crickets and frogs in cyclic pursuit", Technion-Israel Institute of Technology, Haifa, Israel Center for Intelligence Systems Technical Report 9105, July1991.

[14] J.A. Marshall, M.E. Broucke, B.A. Francis: "Formation of vehicles in cyclic pursuit", IEEE Transactions on Automatic Control, Vol 49, No 11, 2004, pp. 1963-1974.

[15] Z. Lin, B.A. Francis, M. Maggiore: "Necessary and sufficient graphical conditions for formation control of unicycles", IEEE Transactions on Automatic Control, Vol 50, No 1, 2005, pp. 121-127.

[16] R.T. Rockafellar: Convex Analysis, Princeton University Press, 1972. 